\title{
Predictive Control Adapting to Fractional Values of Time Delay
}

\author{
Stanislav Talaš $(\mathbb{D}$ and Vladimír Bobál \\ Department of Process Control, Faculty of Applied Informatics, Tomas Bata University in Zlín, Zlín, Czech Republic
}

Correspondence should be addressed to Stanislav Talaš; talas.stanislav@gmail.com

Received 29 June 2017; Accepted 18 March 2018; Published 22 April 2018

Academic Editor: Renming Yang

Copyright (C) 2018 Stanislav Talaš and Vladimír Bobál. This is an open access article distributed under the Creative Commons Attribution License, which permits unrestricted use, distribution, and reproduction in any medium, provided the original work is properly cited.

\begin{abstract}
A combination of identification and control procedures is presented which is aimed at precise control of systems with any value of time delay. Suggested strategy allows the predictive controller to adapt its parameters to a value of the time delay identified during the control process. The system flexibility resides in the ability to work precisely even with time-delay values that are not integer multiples of the sampling period. Overall, the designed approach presents a more precise method to control systems with both static and variable cases of time delay.
\end{abstract}

\section{Introduction}

Time delay is an accompanying effect for a large number of controlled processes not only in industrial areas. Control design for such cases requires an unconventional approach due to its negative impact on stability in traditional control systems. While applying control techniques in discrete form it is necessary to take into account the fact that time delay can be under these circumstances expressed only as an integer multiple of the sampling period. As a result of this limitation, the control precision is comparatively reduced. This article aims to overcome this drawback.

A number of methods is being used to identify the timedelay value in practice; an overview of several was studied in [1]. Among often used concepts there is a comparison of estimates of system outputs for a range of possible timedelay values [2], or also an application of the least square method often combined with identification of all remaining system parameters [3]. Contrary to methods attempting to determine both parameters and time delay, for example, [4, 5], the approach described in this article is focused exclusively on the value of time delay with an assumption that the remaining system parameters are known and constant.

Considering the area of discrete control methods, if we want to perform online measurements of varying timedelay, we need to take into account all changes that may happen between values quantified to individual sampling periods. This problem is still largely studied resulting in number of scientific articles with varying areas of focus. An approach similar to the method described in the article by using specific system parameters and output estimation was taken by [6]. An application of specific mathematical models to specific input signals and performing identification procedure in order to determine individual system parameters were suggested. The control issue of applying model predictive controller to handle time delay was explored in [7] among many others. The mentioned research compared performance of the model-based predictive control (MPC) in state-space system with variable time delay to PI (proportional integrating) controller and Smith predictor, proving efficiency of the predictive approach.

Several procedures exist that are able to control systems with time delay; the most significant are based on principles of the Smith predictor and model based predictive control [8]. Despite their number, only a minority of controllers are sufficiently robust in relation to the value of time delay [9]. Therefore, it is usually suitable to accompany the system control algorithm with an identification method.

The aim of this paper was to explore the option of adapting the control algorithm for cases, when a system changes its time-delay value. Both the concept of time-delay identification and controller adaptation technique were realized in simulations. The first section is focused on connecting individual elements of the control structure. The following 
subchapter analyses the applied method for identification of the time-delay value. The next part investigates specific modifications of the predictive controller in order to incorporate the possibility of controlling a system with time-delay value that is not an exact multiple of the sampling period. Resulting performance of the described control structure is analyzed in the subsequent chapter, followed by final evaluation.

\section{Methods}

The suggested control structure contains a combination of an identification procedure and a control mechanism. Individual sections are realized and connected in the simulation environment MATLAB with the extension Simulink. The connection is designed in such a way that measured values of control input and system output are processed by the time-delay identifying algorithm, first. Consequently, the identification result is provided to the controller, which modifies its own parameters based on acquired data and performs system inputs.

The solution also utilizes MPC principle, where, in contrast to conventional control, the controller output is realized by minimizing proper (usually quadratic) objective function. It is realized as a closed-loop control system. MPC can be realized as system based on input-output process model, as well as state-space theory. In case of this publication the MPC method is based on input-output system model; therefore it utilizes external input-output signals.

2.1. Time-Delay Identification. The foundation of the identification procedure is a method capable of determining an arbitrary value of time delay during the control procedure [10]. This strategy is based on a reversed mechanism of a system behaviour prediction. Therefore, instead of determining system outputs from known parameters, the parameter is deduced from measured outputs. This approach works, provided that the dynamical properties are known and the single unknown value is time delay.

An area of input and output data is reserved for a precise determination of time delay. Based on initial conditions in the section and the series of input signals related to a value of time delay, an estimation of possible system outputs is calculated. As a result, a set of system outputs for possible values of time delay is obtained. Furthermore, individual calculated sequences of outputs are compared with the actual measured one. Comparison of these results enables determining the area of the most probable time-delay value. In addition, the modified $Z$-transform is applied to derive a new variant of the original system parameters for further precision increase. The new version has several times smaller sampling period and its parameters are applied to perform an estimation of system outputs with time delay of a fractional value.

Figure 1 illustrates a comparison between the measured output signal and possible outputs depending on time delay. The red line follows the development of the real system output, blue lines are estimated from the output for four different integer values of time delay and green lines fill the area of possible outputs from estimates computed with fractional time-delay values. The area with increased accuracy

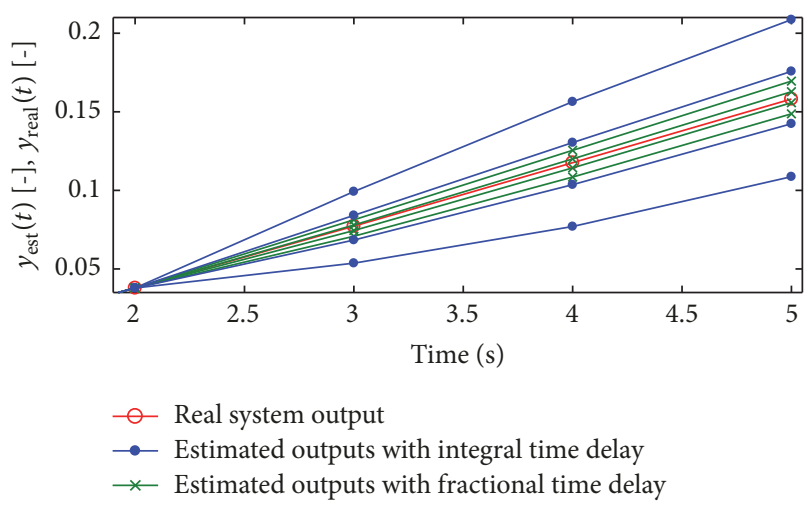

FIGURE 1: Output estimation for various values of time-delay: integral, fractional, and real.

may be determined from the comparison between the integer estimates and the real output first. Therefore, the number of necessary calculations can be significantly decreased and, therefore, even a wide interval of possible time-delay values does not have a major influence on computation demands. The number of estimated outputs can be lowered even further by performing computations for only selected values of time delay as each estimate-comparison operation provides a more accurate information about the real value of time delay in an optimizing search for the minimal deviation.

The comparison between individual calculated output estimates $\hat{y}_{d}$ and the measured data $y_{\text {real }}$ is realized by computing a qualitative criterion of integrated square error (ISE). Its noncontinuous version is expressed by the following equation.

$$
\operatorname{ISE}_{d}=\sum_{i=1}^{n}\left[y_{\text {real }}(i)-\widehat{y}_{d}(i)\right]^{2} .
$$

The most probable value of the time delay is deduced from the sequence with the smallest calculated error. In order to increase the precision for cases with a varying value of time delay, it is possible to incorporate a directional forgetting, which decreases the significance of data measured in more distant areas. As a result, newer measurements have a greater impact.

This identification method enables us to determine the size of time delay directly during the control procedure. Another benefit of this technique is the option to set the amount of studied data. A larger measured area provides more precise results in the presence of disturbance; on the other hand, smaller area may identify a change in the time delay faster.

2.2. Predictive Control Principle. The model based predictive control is a control approach using a mathematical description of the controlled system to estimate its future behaviour. Based on the estimation, an optimal sequence of control inputs is calculated by an optimization algorithm $[11,12]$. The optimality of the desired state is expressed in an objective function with the general form as follows. 


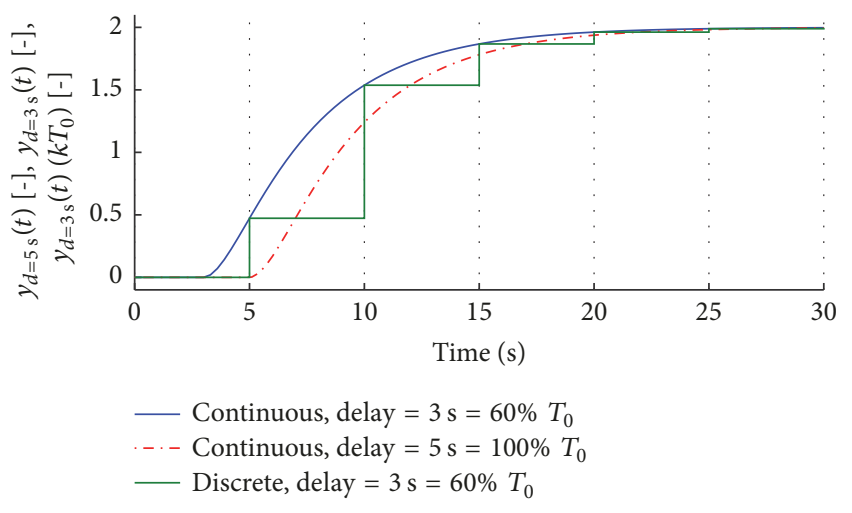

FIGURE 2: System transfer with delay outside of sampling period expressed in discrete area.

$$
\begin{aligned}
J= & \sum_{i=N_{1}}^{N_{2}} \delta(i)[\hat{y}(k+i)-w(k+i)]^{2} \\
& +\sum_{i=1}^{N_{u}} \lambda(i)[\Delta u(k+i-1)]^{2},
\end{aligned}
$$

Variables $\delta$ and $\lambda$ represent weighting parameters determining the balance between the smallest control error and the slowest change in control input. The area of optimization is determined by horizons $N_{1}, N_{2}$, and $N_{u}$ which set the number of sampling steps involved in calculations. The goal of optimization is to find a series of input signal differences $\Delta u$ that lead to the smallest value of $J$.

Only the first value of the optimized series is applied as $\Delta u(k)$ and the entire procedure is repeated in the following sampling step; this is called receding horizon strategy.

The applied controller was based on the traditional design of the generalized predictive controller (GPC). This method is popular due to its versatility. The controlled system dynamics are represented by a transfer function as a base for the future output estimation. The benefit lies in its principle that it uses only a small number of values in order to reconstruct the entire step function or any output shape with knowledge of the input signal [13-16].

2.3. Modification of the Predictive Control Procedure. The control procedure is based on the above described GPC controller, in which sections of system model and future output predictions are modified.

A system described in the discrete area has the timedelay parameter represented by the power of variable $z^{-d}$. The value of $d$ can assume only integral values, and, therefore, it is necessary to describe such system with modified $Z$ transform, which can express system dynamic outside of the original sampling area.

Figure 2 displays how a system can be described in discrete area with such a time delay that is not an integer multiple of its sampling period. The mathematical description starts in a model without time delay, from which the system state in the fraction of sampling period given by the length of time delay is deduced. The value of system response with the given length of time delay then represents the state achieved in the moment of sampling. As the source for determining the controller parameters is used a system with time-delay value rounded down and the remaining parameters were obtained from the modified $Z$-transformation.

Based on parameters received by modified $Z$-transform, it is necessary to deduce new values of matrices applied in the predictive control. During the estimation of future outputs based on a system expressed as a division of polynomials, where the order of numerator is $n b$ and the order of denominator is $n a$, the prediction of the output variable is given by the following equation:

$$
\widehat{y}(k+1)=\sum_{i=1}^{n a+1} a_{i} y(k+1-i)+\sum_{i=1}^{n b} b_{i} \Delta u(k-d-i) .
$$

With the prediction of length of $N$ sampling steps and with integral part of delay of size $d$, individual elements are divided according to their affiliation to the following vectors:

$$
\begin{gathered}
u=\left[\begin{array}{c}
\Delta u(k) \\
\Delta u(k+1) \\
\vdots \\
\Delta u(k+N-1)
\end{array}\right], \\
u_{1}=\left[\begin{array}{c}
\Delta u(k-1) \\
\Delta u(k-2) \\
\vdots \\
\Delta u(k-n b)
\end{array}\right] \\
y_{1}=\left[\begin{array}{c}
\hat{y}(k+d) \\
\hat{y}(k+d-1) \\
\vdots \\
\hat{y}(k+d-n a)
\end{array}\right]
\end{gathered}
$$

And the result is a matrix form interpreted as

$$
\widehat{\mathbf{y}}=\mathbf{G u}+\mathbf{H u}_{1}+\mathbf{S y}_{1} .
$$

Considering the fact that the system obtained by the modified $Z$-transformation contains a nonzero element $b_{0}$, it is required to fill additional parameters to matrices $\mathbf{G}$ and $\mathbf{H}$.

In the presence of the time delay, it is necessary to estimate future system outputs to determine the entire vector $y_{1}$. The equation for calculating these values (3) is another section that has to be altered based on system model. Besides the obvious change in existing parameters, the variable $b_{0}$ needs to be added.

Figure 3 shows the layout of the control scheme. The control input signal $u$ originating from the predictive controller and the output signal $y$ measured in the controlled system are recorded in every sampling step by the time-delay identification algorithm. The newly recoded data is added to the set of known values and the identification procedure 


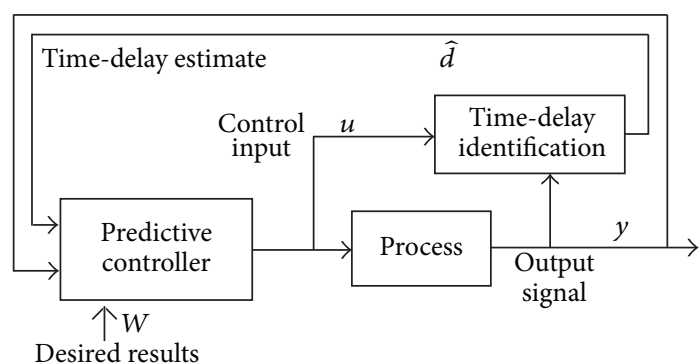

FIgURE 3: Adaptive control scheme.

performs a new estimate $\widehat{d}$ of the time delay between the control input and the output signal. The result is consequently provided back to the predictive controller, which modifies its internal parameters accordingly. The whole procedure is ideally performed as a part of controller computations to ensure a fast response to sudden changes in time delay.

The result of the above-mentioned operations is a form of predictive control, which is able to adapt to a different dynamic caused by fractional value of time delay, while maintaining the original sampling period.

\section{Results}

The design functionality was verified in the simulation environment MATLAB with the Simulink extension. The system used for simulation was selected for its simple dynamics. The main goal was to demonstrate the ability of the controller to adapt itself to varying conditions of time delay.

$$
G(s)=\frac{2}{4 s^{2}+5 s+1} .
$$

In the discrete version with the sampling period of $2 \mathrm{~s}$,

$$
G\left(z^{-1}\right)=\frac{0.4728 z^{-1}+0.2076 z^{-2}}{1-0.7419 z^{-1}+0.08208 z^{-2}} .
$$

The development of the time-delay value was selected in such form to involve both fluent changes and sudden steps. The reference trajectory was set with respect to the fact that the method of time-delay identification needs to measure varying changes to properly determine the system state.

The predictive controller GPC had its weighting parameters set to focus more on the precision of the output signal in ratio of $1: 2$.

Figure 4 contains results of individual approaches aiming to provide information about the development of time-delay value represented by the black dotted line. The first signal uses the method proposed in the article identifying the delay with the accuracy on tenths of a second. The algorithm is able to closely follow the real value with the exception in the interval from $220 \mathrm{~s}$ to $240 \mathrm{~s}$ where the output signal remains constant. Therefore, the delay cannot be determined and maintains the last known value of 3.8 sampling period.

The second signal is a result of time-delay identification with accuracy to integers. The control precision is decreased in critical areas, where the real system time delay is distant from integral value, for example, from the time of $250 \mathrm{~s}$ up to the $300 \mathrm{~s}$.

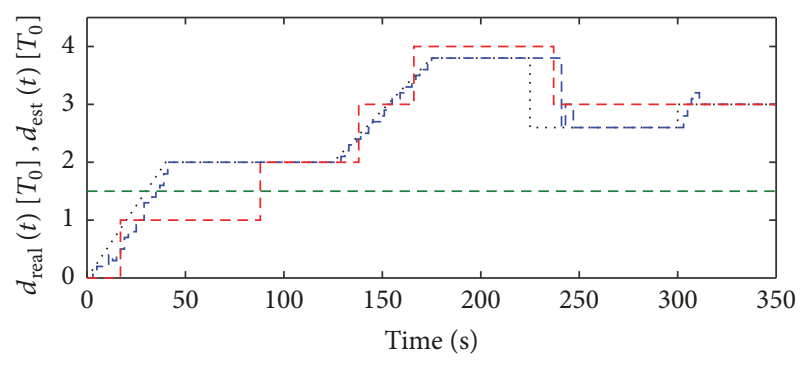

$\begin{array}{ll}\text {...... Real delay } & --- \text { Integer delay estimates } \\ \text { - - - Fractional delay estimates } & --- \text { Average delay estimate }\end{array}$

FIGURE 4: Time-delay estimates for the adaptive control.

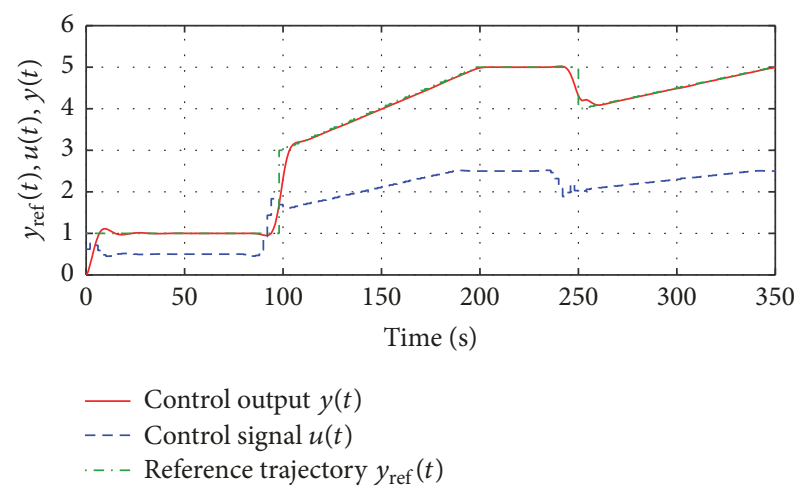

FIGURE 5: System control adapting to a varying time delay.

The third signal is a constant value of time delay over the entire control process selected experimentally to avoid risks of instability in areas with large differences from the estimate, for example, at the start of the process and from $175 \mathrm{~s}$ to $225 \mathrm{~s}$.

Figure 5 demonstrates how with the changing value of time delay the control input $u$ is also adapting in order to reach the reference trajectory $y_{\text {ref }}$. The controller obtains the estimated time-delay value from the identification algorithm and, based on this value, it changes parameters in control matrices, which are used in calculation of the control input. Therefore, the controller is able to adapt to a change of time delay in the form of fluent change in area $125 \mathrm{~s}$ to $175 \mathrm{~s}$, as well as during step changes at $300 \mathrm{~s}$. The relation between the identification and the control works as a mutually complementary mechanism. Change in the control input is eventually followed by a response in the output signal. The time difference in this relation is measured by the identification algorithm which provides the controller with proper data. The less precise the initial estimate of the controller is, the more significant the control input is required leading to a greater accuracy in the time-delay estimation and in the overall control as a result.

The amount of contribution of the designed identification method is demonstrated on Figures 6 and 7, where a certain level of control functionality has been limited.

Figure 6 shows the variant in which the control system is adapting to the time delay, which is being identified with accuracy of integers. The control precision has decreased in 


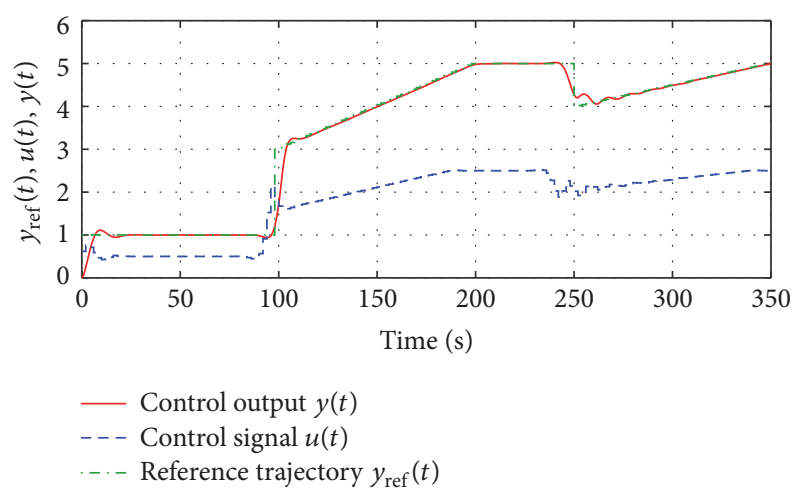

FIGURE 6: System control with time delay identified with accuracy of integers.

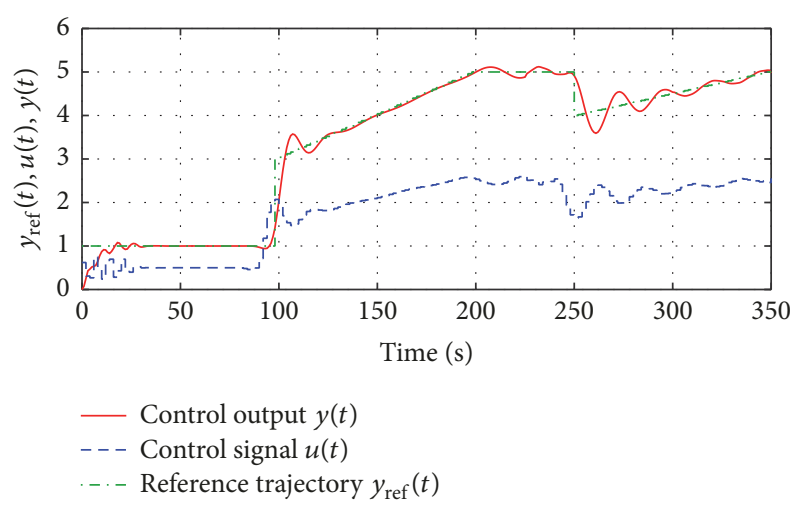

FIGURE 7: System control with constant estimated value of time delay.

TABLE 1: Comparison of the quality for various versions of timedelay identification.

\begin{tabular}{lc}
\hline Time-delay estimation & ISE criterion value \\
\hline Constant delay $1.5 \cdot T_{0}$ & 16.2638 \\
Identification of integral time delay & 13.1859 \\
Identification of fractional time delay & 7.7763 \\
\hline
\end{tabular}

critical areas, where the real system time delay is distant from integral value, for example, around $250 \mathrm{~s}$.

Figure 7 illustrates the situation when the estimated time delay is represented by a constant value. The precision is heavily affected by the current distance between the real value and the constant. The negative effects are mainly visible at times of step response, but during the fluent change it is easier for the controller to adapt.

From Table 1 it is apparent that the designed combination of identification and control provides a significant improvement in the quality of systems that change the value of time delay during the control procedure.

\section{Conclusion}

The article describes new procedures for adaptive control of systems with variable time delay. The control structure is based on mutually cooperating algorithms fulfilling the function of time-delay identification on one hand and system output regulation on the other.

Time-delay identification is performed based on the knowledge of dynamical parameters of the controlled system, when the section of data measured in the past is compared to estimation of output development for a range of possible time-delay values. The accuracy of this approach is expanded by incorporating system model parameters with decreased sampling period and therefore it is able to operate with a greater accuracy than just individual lengths of the original sampling period.

The control procedure is founded on the traditional concept of GPC, which is extended with a function to predict behaviour of the system, where the time delay is a fractional multiple of the sampling time. Parameters, defining the system dynamic, are altered by modified $Z$-transform, which provides information about the system state outside of the original sampling area. Prediction matrices and output estimation are changed based on these parameters, in order to provide output predictions for systems with fractional time delay.

Functionality of the design was verified by simulations. The time-delay identification mechanism has managed to determine the size of the real time delay with a great accuracy and quickly react to its changes. The control algorithm with matrices modifiable by system model with a fractional time delay has succeeded to adequately adapt to varying conditions. The overall combination of these methods creates a chance of precise control mainly for systems with risk of unpredictable variations in the time-delay value during the control procedure.

Despite the presented functionality the described approach contains several possible directions of further improvement. One of them is the robustness of the identification procedure that would decrease its sensitivity to inaccuracies in estimated system parameters. Another option could be incorporating an identification of system parameters that were to this point considered known and constant.

\section{Conflicts of Interest}

The authors declare that there are no conflicts of interest regarding the publication of this paper.

\section{Acknowledgments}

This work was supported by Internal Grant Agency of Tomas Bata University under Project no. IGA/FAI/2017/009.

\section{References}

[1] J.-P. Richard, "Time-delay systems: an overview of some recent advances and open problems," Automatica, vol. 39, no. 10, pp. 1667-1694, 2003.

[2] S. V. Drakunov, W. Perruquetti, J.-P. Richard, and L. Belkoura, "Delay identification in time-delay systems using variable structure observers," Annual Reviews in Control, vol. 30, no. 2, pp. 143-158, 2006. 
[3] A. Elnaggar, G. A. Dumont, and A.-L. Elshafei, "Recursive estimation for system of unknown delay," in Proceedings of the 28th IEEE Conference on Decision and Control. Part 2 (of 3), pp. 1809-1810, December 1989.

[4] R. B. C. Lima and P. R. Barros, "Identifiaction of time-delay systems: a state-space realization approach," in Proceedings of the 9th IFAC Symposium on Advnced Control of Chemical Processes ADCHEM, pp. 254-259, 2015.

[5] A. Atitallah, S. Bedoui, and K. Abderrahim, "An Optimal Two Stage Identification Algorithm for Discrete Hammerstein Time Delay Systems," IFAC-PapersOnLine, vol. 49, no. 10, pp. 19-24, 2016.

[6] C. Shah, "On the modelling of time-varying delays," Tech. Rep., AM University, Texas, 2004.

[7] S. Himpe and V. Theunynck, Design and advanced control of a process with variable time delay, University of Ghent, 2006.

[8] J. E. Normey-Rico and E. F. Camacho, "Control of Dead-Time Processes," IEEE Control Systems Magazine, vol. 28, no. 5, pp. 136-137, 2008.

[9] E. Normey-Rico and E. F. Camacho, "Dead-time compensators: a survey," Control Engineering Practice, vol. 16, no. 4, pp. 407428, 2008.

[10] S. Talaš, V. Bobál, A. Krhovják, and L. Rušar, "Discrete method for estimation of time-delay outside of sampling period," in Proceedings of the 30th European Conference on Modelling and Simulation, ECMS 2016, pp. 287-292, deu, June 2016.

[11] D. W. Clarke, C. Mohtadi, and P. S. Tuffs, "Generalized predictive control-Part I. the basic algorithm," Automatica, vol. 23, no. 2, pp. 137-148, 1987.

[12] D. W. Clarke, C. Mohtadi, and P. S. Tuffs, "Generalized Predictive Control-Part II Extensions and interpretations," Automatica, vol. 23, no. 2, pp. 149-160, 1987.

[13] J. M. Maciejowski, Predictive Control with Constraints, Prentice Hall, London, UK, 2002.

[14] J. A. Rossiter, Model Based Predictive Control: a Practical Approach, CRC Press, 2003.

[15] E. F. Camacho and C. Bordons, "Nonlinear model predictive control: an introductory review," in Assessment and future directions of nonlinear model predictive control, vol. 358 of Lect. Notes Control Inf. Sci., pp. 1-16, Springer, Berlin, 2007.

[16] V. Bobal, M. Kubalcik, P. Dostal, and J. Matejicek, "Adaptive predictive control of time-delay systems," Computers \& Mathematics with Applications. An International Journal, vol. 66, no. 2, pp. 165-176, 2013. 


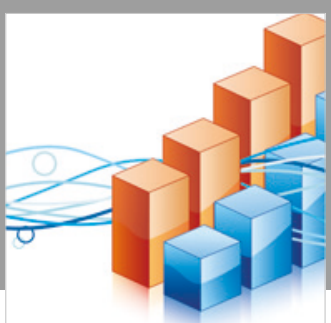

Advances in

Operations Research

\section{-n-m}
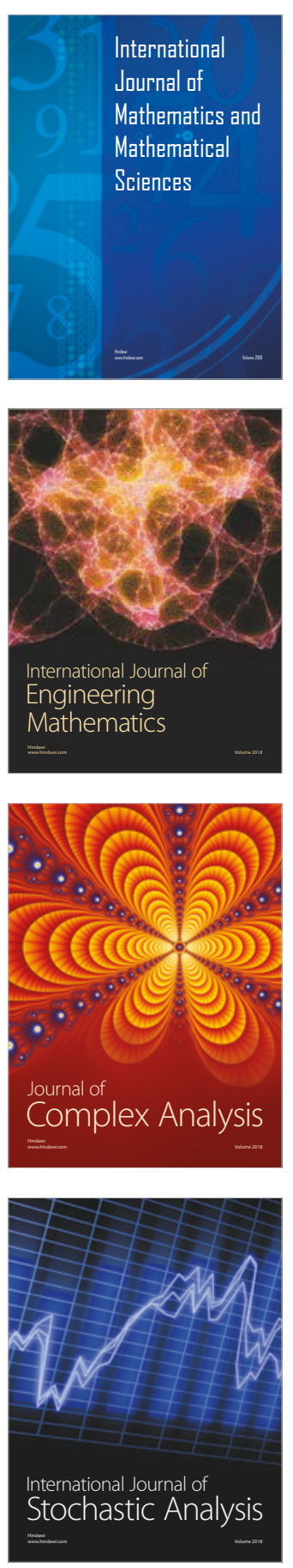
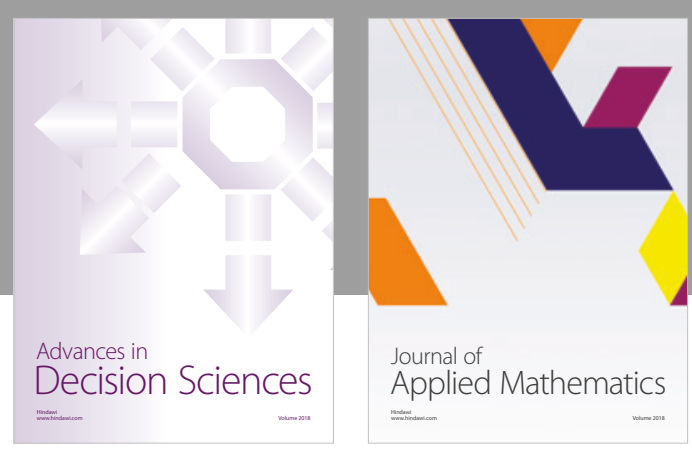

Journal of

Applied Mathematics
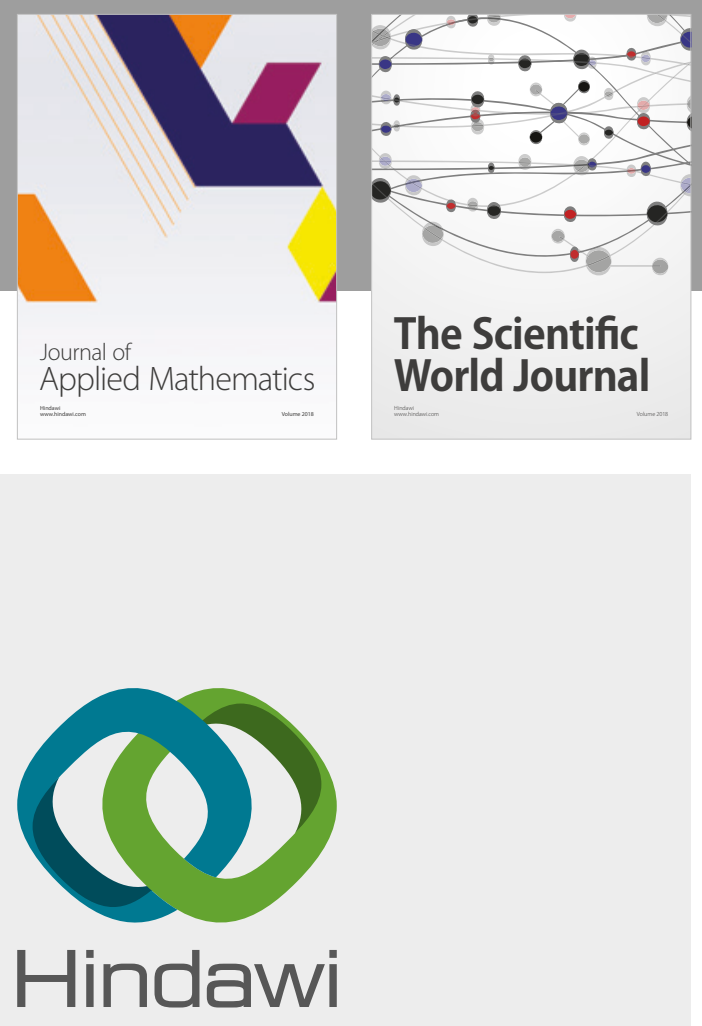

Submit your manuscripts at

www.hindawi.com

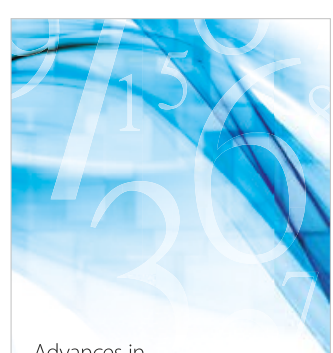

Advances in
Numerical Analysis
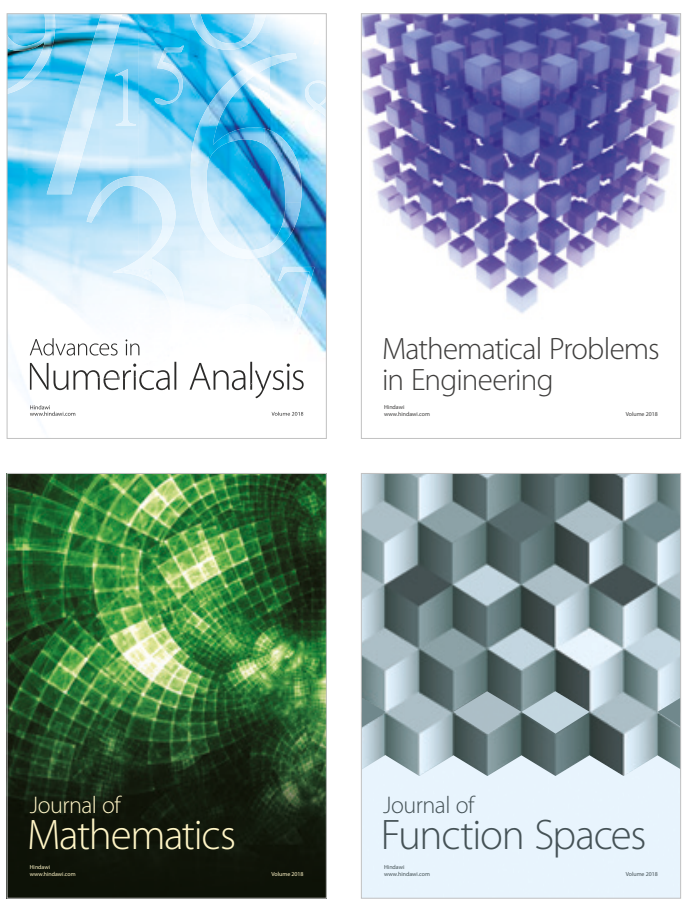

Mathematical Problems in Engineering

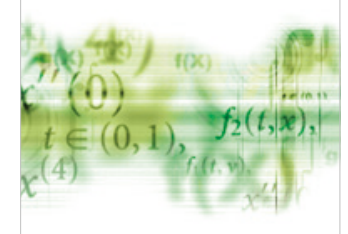

International Journal of

Differential Equations

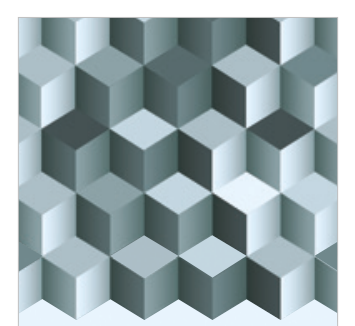

Journal of

Function Spaces

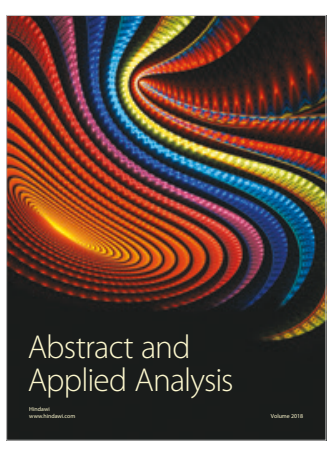

The Scientific

World Journal

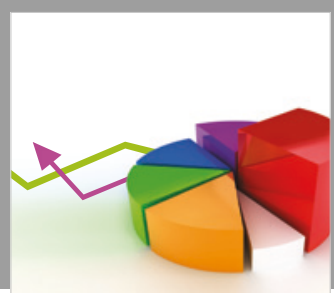

Journal of

Probability and Statistics
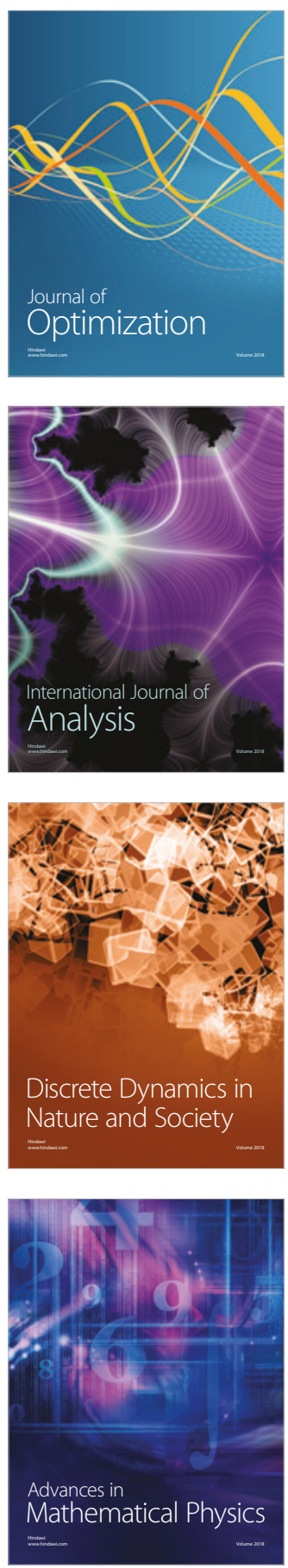